\title{
Complete remission of primary cutaneous anaplastic large cell lymphoma after a short course of brentuximab vedotin
}

\author{
ELISA MILAN $^{1 *}$, PAOLA MICELI $^{1 *}$, ALVISE SERNICOLA $^{1 *}$, SILVIA FINOTTO $^{2}$, \\ DARIO MARINO $^{2}$ and MAURO ALAIBAC ${ }^{1}$ \\ ${ }^{1}$ Unit of Dermatology, University of Padua; ${ }^{2}$ Unit of Medical Oncology 1, \\ Veneto Institute of Oncology IOV-IRCCS, Padova I-35128, Italy
}

Received March 31, 2020; Accepted February 15, 2021

DOI: $10.3892 / \mathrm{mco} .2021 .2283$

\begin{abstract}
Primary cutaneous anaplastic large cell lymphoma (PCALCL) is a rare $\mathrm{CD} 30^{+}$lymphoproliferative disorder characterized by the development of lesions ranging from papules to large tumors. Most cases present as localized disease, however multifocal and generalized involvement of the skin can occur. Several treatments have been proposed for PCALCL; however a highly effective standard approach to multifocal disease has not yet been elucidated. The disease expression of CD30 antigen in at least $75 \%$ of the tumor makes it an optimal target for immunotherapy. The current study presents a case of a 62-year-old male referred to the University of Padua Dermatology Clinic complaining about nodular and ulcerated lesions involving the frontal area and scalp that were $8 \mathrm{~cm}$ in diameter. Doses of $180 \mathrm{mg}$ brentuximab vedotin (BV), which is an antibody drug conjugate binding CD30 antigen, were administered every 21 days. A 75\% decrease in dimensions after the first infusion and a complete remission after the second was observed. Disease response appeared to be dose-related and adverse reactions, in particular peripheral neuropathy, may be an effect of cumulative toxicity, meaning that treatment cycle reduction should be considered. Based on the present results, A high dose, short course of $\mathrm{BV}$ is recommended as a cost-effective approach for PCALCL. However, further studies are required to assess the efficacy and other potential advantages of this therapeutic regimen.
\end{abstract}

\section{Introduction}

Primary cutaneous CD30+ lymphoproliferative disorders (LPDs) are rare diseases but represent the second most frequent form of cutaneous T-cell lymphoma after mycosis fungoides $(1,2)$. According to the 2018 update of the WHO-EORTC classification,

Correspondence to: Professor Mauro Alaibac, Unit of Dermatology, University of Padua, via Gallucci 4, Padova I-35128, Italy

E-mail: mauro.alaibac@unipd.it

${ }^{*}$ Contributed equally

Key words: lymphoproliferative disorder, CD30, brentuximab vedotin, primary cutaneous anaplastic large cell lymphoma, immunotherapy
CD30 ${ }^{+}$T-cell LPDs range from lymphomatoid papulosis (LYP) to primary cutaneous anaplastic large-cell lymphoma (PCALCL) and constitute a spectrum of disorders expressing CD30 antigen in tumor cells $(1,3,4)$. The two conditions feature different presentation and clinical course: LYP is characterized by papulonodular lesions undergoing spontaneous regression over a chronic course of years to decades, while PCALCL manifests with solitary or grouped, rapidly growing and ulcerating tumors or plaques. PCALCL typically affects older males and in $20 \%$ of cases presents multifocal skin involvement. In $30 \%$ of cases, PCALCL tends to spontaneous regression, yet to a lesser extent and duration than LYP (1). Diagnosis is based on histopathology and immunohistochemistry revealing $\mathrm{CD} 30^{+}$large pleomorphic or anaplastic T-cells; in PCALCL at least $75 \%$ of tumor cells are $\mathrm{CD}^{2} 0^{+}(1,3)$. Extra-cutaneous disease spreading should be ruled out with staging procedures including whole body PET-CT scan and, in the case of multifocal tumors, extracutaneous disease and abnormal laboratory exams, bone marrow examination (1). Management of PCALCL should be tailored to each case taking into account the severity of cutaneous involvement and the indolent nature of the disease, with 5-year survival rates between $76-96 \%$ (1). The disease has an intrinsic tendency to skin relapse in $\sim 39 \%$ of patients, associated to a limited risk of systemic spreading in only $13 \%$ of cases (2). In cases of localized disease, a wait-and-see strategy can be appropriate as spontaneous complete remission occurs in up to $22 \%$ of cases after a median period of 2 months (range 1-6 months) $(1,2)$. Surgical excision or local radiation can be additionally considered based on tumor burden (2). The approach to multifocal or disseminate disease should consider that aggressiveness of therapy does not influence the frequency of relapse and that consequently the aim of treatment cannot be sustained remission. Alternatives in disseminate or progressive forms include single cytotoxic drugs, such as methotrexate, or polychemotherapy regimens based on anthracyclines, such as CHOP (3). Brentuximab vedotin (BV) has recently shown promising results in the treatment of PCALCL even if the optimal dosing is currently not defined.

\section{Case presentation}

A 62-year-old Caucasian man was referred to our Dermatology Department complaining the abrupt appearance and quick advancement of cutaneous nodules, associated with a sensation 
of skin pain and tenderness. The patient reported the onset of a single lesion in the right frontal region, progressively increasing in size since June 2019, followed by the appearance of other nodules in the scalp and finally by the rapid development of another nodular left frontal lesion in August 2019. The patient had a personal history of arterial hypertension, gastroduodenal ulcer and bilateral ischemic optic neuropathy in therapy with atenolol, perindopril, indapamide, amlodipine, acetylsalicylic acid and pantoprazole. Clinical examination revealed diffuse nodules on the scalp and on the frontal area, where the two major lesions, $4.5 \mathrm{~cm}$ diameter in the right frontal region (Fig. 1A) and $8 \mathrm{~cm}$ in the left, were observed. The nodular lesions were elevated $\sim 3 \mathrm{~cm}$ from the surrounding skin, firm to touch and presented an ulcerated erythematous surface. The characteristics of lesions and the history of rapid growth were highly suggestive for a cutaneous localization of lymphoproliferative disease.

Multiple biopsy specimens were obtained in September and October, setting a differential diagnosis between LYP and PCALCL. A CT-scan ruled out extra-cutaneous localizations of disease and peripheral blood cytometry showed no abnormal immunophenotypes. Finally, a bone marrow specimen was obtained, and the results proved normal bone cellularity with no signs of cellular atypia and a maintained leuco-erithroblastic ratio.

As soon as the diagnosis was confirmed by histology, we started therapy with methotrexate $7.5 \mathrm{mg}$ sc weekly and prednisone $25 \mathrm{mg}$ po daily. At the follow-up visit, scheduled after one month, we found a size increase in the lesions and we raised the dose of methotrexate up to $15 \mathrm{mg}$ weekly and considered alternative treatments. Because of the clinical extent and aggressive behavior of the disease we decided to start therapy with BV at the dosing of $180 \mathrm{mg}(1.8 \mathrm{mg} / \mathrm{kg})$ every three weeks. Clinical assessment at three weeks from the first infusion of BV showed an important decrease in volume of lesions, with the prominent left frontal reduced to $3.5 \mathrm{~cm}$ and the right one to less than $1 \mathrm{~cm}$ in diameter. Two weeks after the second infusion, we appreciated a complete remission of all skin lesions with only mild hyperpigmented and fibrotic results (Fig. 1B).

The patient is scheduled for 16 infusions of brentuximab $180 \mathrm{mg}$ every three weeks, four of which have been already administered. The disease is in complete remission and the patient complained no adverse events following treatment. Monthly monitoring of laboratory tests before and during treatment showed no abnormal values.

\section{Discussion}

Currently, there is no standard therapeutic approach for cases of multifocal PCALCL. Combination chemotherapy has been frequently administered for multifocal primary cutaneous disease, even though no specific regimen has been reported as superior and doxorubicin-based combinations, such as CHOP (cyclophosphamide, doxorubicin, vincristine and prednisone), are more frequently used. On the basis of a review by Shehan et al relapses seem to occur more frequently in patients receiving traditional chemotherapy and this result doesn't correlate with a more aggressive treatment of extensive disease (3). Moreover, the Dutch Cutaneous Lymphoma Group

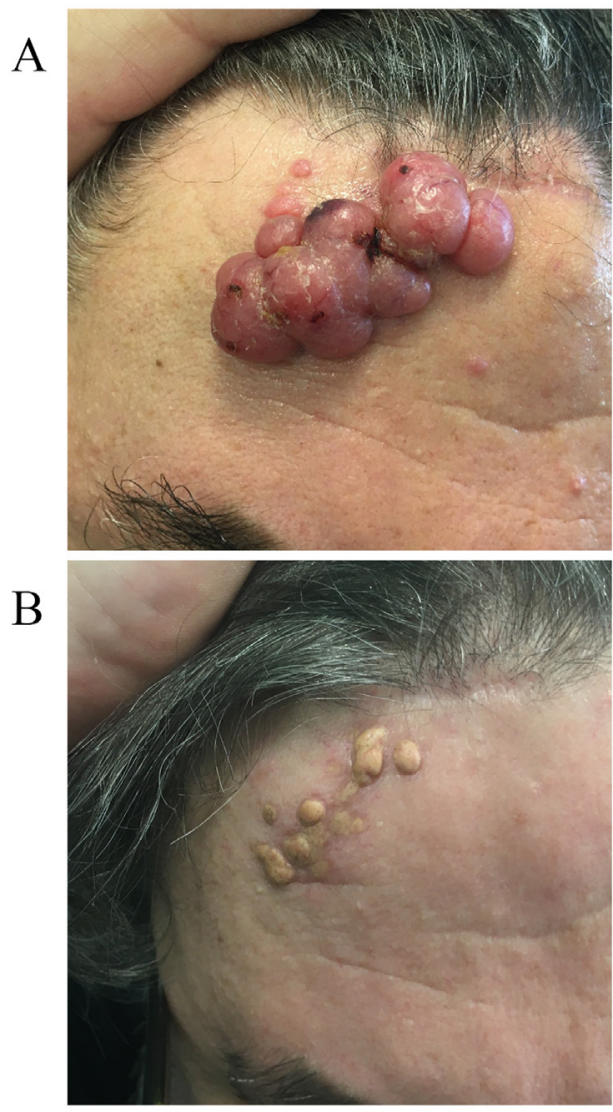

Figure 1. Primary cutaneous anaplastic large cell lymphoma. (A) Nodular lesions on the right frontal region at baseline and (B) at clinical reassessment two weeks after the second infusion of brentuximab vedotin.

found that all patients treated with $\mathrm{CHOP}$ regimen developed one or more relapses in the skin and authors suggested not to use traditional chemotherapy for multifocal ALCL involving the skin (5). Radiotherapy or low-dose methotrexate, that was initially administered to our patient, have also been proposed as first-line treatment for multifocal or relapsing PCALCL limited to skin $(5,6)$. Moreover, systemic retinoids including bexarotene, interferon alpha- $2 \mathrm{a}$ and thalidomide have been effective treatments for multifocal PCALCL (1). Etoposide monotherapy and autologous bone-marrow transplantation have also been proposed for relapsed multifocal disease (3).

Finally, BV is an antibody-drug conjugate (ADC) that combines a chimeric anti-CD30 antibody to the anti-microtubule agent monomethyl auristatin E (MMAE) $(7,8)$. Upon binding to $\mathrm{CD} 30$ receptor, the ADC is internalized by endocytosis and undergoes consequent lysosomal degradation allowing MMAE to bind microtubules and cause cell cycle arrest and apoptosis (7). CD30 is a cell surface leukocyte antigen constituted by a type I transmembrane glycoprotein and an extracellular domain homologous to tumor necrosis factor and nerve growth factor receptor family members. CD30 is normally expressed in activated T, B, and natural killer lymphocytes. Certain LPDs (including Hodgkin lymphoma, ALCL, CTCL, a fraction of diffuse large B-cell lymphomas and of follicular lymphomas) and Kaposi sarcoma express the CD30 antigen as well $(2,9)$. The function of CD30 has not been defined yet, but it seems implicated in both cell death and proliferation (9). Targeted delivery of MMAE to 
CD30 expressing tumor cells makes BV a well-suited target for immunotherapy. Based on our observation of significant disease response after the first BV infusion and complete remission after the second one, we suggest that high-dose short course therapy with BV could be an appropriate approach for PCALCL. While therapeutic management of Hodgkin lymphoma (HL) with BV is better established and more clinical data are available, PCALCL treatment protocol is not yet well defined. BV is approved by the FDA for both HL and ALCL at an intravenous dose of $1.8 \mathrm{mg} / \mathrm{kg}$ every 3 weeks (7). While treatment is usually administered for a maximum of 16 cycles or until either disease progression or unacceptable toxicity occur, multiple studies have been conducted in recent years with different dosing regimens of BV (7). Fanale et al in a phase I study with CD30 ${ }^{+}$LPDs administered a $0.4-1.4 \mathrm{mg} / \mathrm{Kg} /$ dose weekly for 3 weeks every 28 days obtaining an overall response rate (ORR) of $59 \%$ and a complete response (CR) of $34 \%$ (10). A phase II study of BV in PCALCL enrolled 11 patients receiving six doses each of $0.4-1.2 \mathrm{mg} / \mathrm{kg}$, achieving an ORR of $82 \%$ and a CR in $55 \%$ of cases (9). Though limited, these results support the hypothesis that clinical response to BV in PCALCL could be dose-related and suggest use of maximal dosing equivalent to $1.8 \mathrm{mg} / \mathrm{kg}$. Moreover, the fast response observed in our case report could be explained by the high percentage of $\mathrm{CD} 30^{+}$cells present in PCALCL on which the drug exerts a targeted mechanism of action. PCALCL expresses CD30 antigen in at least $75 \%$ of tumor mass, thus implicating that after each cycle of BV $\sim 75 \%$ of cells die as a result of direct killing. In addition, such a rapid dimensional decrease could be the result of an indirect action of BV. In fact, MMAE produce a well-known toxic effect by diffusing into surrounding stroma, destroying not only cells internalizing the ADC but also proximal tumor cells (7). Furthermore, our patient experienced a quick reduction in lesion size after the first dose, which is consistent with the pharmacokinetics of MMAE gaining maximum concentration approximately 1-3 days post infusion (7).

High dose protocols over a short course may be associated with a beneficial safety profile. Therapy with BV has been related to toxicities mostly of grade 1 or 2 . The most common adverse reactions, observed in $>20 \%$ of cases, have been peripheral sensory neuropathy, fatigue, diarrhea, neutropenia, vomiting, pyrexia, anemia, upper respiratory tract infections, fever, thrombocytopenia $(7,11)$. However, serious adverse reactions such as peripheral motor neuropathy, septic shock, supraventricular arrhythmia, progressive multifocal leukoencephalopathy and one case of Steven-Johnson syndrome were reported in phase II trials (7). Peripheral neuropathy is the most common side effect, experienced by $55 \%$ of patients, responsible for treatment discontinuation in $12 \%$ of cases and for dose-reduction in $10 \%$ (7). Moreover, this side effect seems to be more common when the drug is administered weekly rather than every three weeks. Younes et al reported $22 \%$ of patients experiencing peripheral neuropathy with $1.8 \mathrm{mg} / \mathrm{kg}$ every 3 weeks of BV compared to $66 \%$ of cases described by Fanale et al with a weekly dose of 0.4-1.4 mg/kg $(10,12)$. Although usually reversible, peripheral neuropathy is typically an effect of cumulative toxicity, thus encouraging reduction in number of cycles and frequency of administration (7).
In the end, considering an estimate cost of 200.000 euros for 16 cycles of BV in a patient of $80 \mathrm{~kg}$, a short cycle may considerably improve cost-effectiveness (13).

In conclusion, PCALCL has a generally indolent behavior even in disseminated presentations and shows frequent cutaneous relapses that maintain responsiveness to treatments. Well-designed clinical trials are needed to determine the efficacy of high dose short course therapy with BV and to assess whether there are other potential advantages over standard 16 cycle protocol.

\section{Acknowledgements}

Not applicable.

\section{Funding}

No funding was received.

\section{Availability of data and materials}

The datasets used and/or analyzed during the current study are available from the corresponding author on reasonable request.

\section{Authors' contributions}

EM, PM and AS prepared the manuscript. EM and PM performed the literature analysis search. AS, MA, SF and DM conceived and designed the current study. AS and MA drafted and critically revised the manuscript for important intellectual content. AS prepared the figures. MA gave final approval of the version to be published. All authors read and approved the final manuscript. MA and AS confirm the authenticity of all the raw data.

\section{Ethics approval and consent to participate}

Not applicable.

\section{Patient consent for publication}

Written informed consent for publication of their clinical details and clinical images was obtained from the patient.

\section{Competing interests}

The authors declare that they have no competing interests.

\section{References}

1. Kempf W, Pfaltz K, Vermeer MH, Cozzio A, Ortiz-Romero PL, Bagot M, Olsen E, Kim YH, Dummer R, Pimpinelli N, et al: EORTC, ISCL, and USCLC consensus recommendations for the treatment of primary cutaneous CD30-positive lymphoproliferative disorders: Lymphomatoid papulosis and primary cutaneous anaplastic large-cell lymphoma. Blood 118: 4024-4035, 2011.

2. Liu HL, Hoppe RT, Kohler S, Harvell JD, Reddy S and Kim YH: $\mathrm{Cd} 30^{+}$cutaneous lymphoproliferative disorders: The stanford experience in lymphomatoid papulosis and primary cutaneous anaplastic large cell lymphoma. J Am Acad Dermatol 49: 1049-1058, 2003.

3. Shehan JM, Kalaaji AN, Markovic SN and Ahmed I: Management of multifocal primary cutaneous $\mathrm{CD} 30^{+}$anaplastic large cell lymphoma. J Am Acad Dermatol 51: 103-110, 2004. 
4. Willemze R, Cerroni L, Kempf W, Berti E, Facchetti F, Swerdlow SH and Jaffe ES: The 2018 update of the WHO-EORTC classification for primary cutaneous lymphomas. Blood 133: 1703-1714, 2019.

5. Bekkenk MW, Geelen FA, van Voorst Vader PC, Heule F, Geerts ML, van Vloten WA, Meijer CJ and Willemze R: Primary and secondary cutaneous CD30(+) lymphoproliterative disorders: A report from the Dutch Cutaneous Lymphoma Group on the long-term follow-up data of 219 patients and guidelines for diagnosis and treatment. Blood 95: 3653-3661, 2000.

6. Artemi P, Wong D, Mann S and Regan W: CD30 (Ki-1)-positive primary cutaneous T-cell lymphoma: Report of spontaneous resolution. Australas J Dermatol 38: 206-208, 1997.

7. Minich SS: Brentuximab vedotin: A new age in the treatment of hodgkin lymphoma and anaplastic large cell lymphoma. Ann Pharmacother 46: 377-383, 2012.

8. Donato EM, Fernández-Zarzoso M, Hueso JA and de la Rubia J: Brentuximab vedotin in hodgkin lymphoma and anaplastic large-cell lymphoma: An evidence-based review. Onco Targets Ther 11: 4583-4590, 2018.

9. Duvic M, Reddy SA, Pinter-Brown L, Korman NJ, Zic J, Kennedy DA, Lorenz J, Sievers EL and Kim YH: A phase II study of SGN-30 in cutaneous anaplastic large cell lymphoma and related lymphoproliferative disorders. Clin Cancer Res 15: 6217-6224, 2009.
10. Fanale MA, Forero-Torres A, Rosenblatt JD, Advani RH, Franklin AR, Kennedy DA, Han TH, Sievers EL and Bartlett NL: A phase I weekly dosing study of brentuximab vedotin in patients with relapsed/refractory CD30-positive hematologic malignancies.Clin Cancer Res 18: 248-255, 2012.

11. Gravanis I, Tzogani K, van Hennik P, de Graeff P, Schmitt P, Mueller-Berghaus J, Salmonson T, Gisselbrecht C, Laane E, Bergmann L, et al: The European medicines agency review of brentuximab vedotin (Adcetris) for the treatment of adult patients with relapsed or refractory $\mathrm{CD} 30^{+}$hodgkin lymphoma or systemic anaplastic large cell lymphoma: Summary of the scientific assessment of the committee. Oncologist 21: 102-109, 2016.

12. Younes A, Bartlett NL, Leonard JP, Kennedy DA, Lynch CM, Sievers EL and Forero-Torres A: Brentuximab vedotin (SGN-35) for relapsed CD30-positive lymphomas. N Engl J Med 363: 1812-1821, 2010.

13. Prince HM, Kim YH, Horwitz S, Dummer R, Scarisbrick J, Quaglino P, Zinzani PL, Wolter P, Sanches JA, OrtizRomero PL, et al: Brentuximab vedotin or physician's choice in CD30-positive cutaneous T-cell lymphoma (ALCANZA): An international, open-label, randomised, phase 3, multicentre trial. Lancet 390: 555-566, 2017. 